\title{
A INVESTIGAÇÃO INTERAMERICANA DE MORTALIDADE NA INFÂNCIA EM SÃO PAULO, BRASIL
}

\author{
COMUNICAÇÃO
}

Ruy LAURENTI (1)

LAURENTI, R. - A investigação interamericana de mortalidade na infância, em São Paulo, Brasil. Rev. Saúde públ., S. Paulo, 3(2):225-229, dez. 1969.

\begin{abstract}
Resumo - As taxas de mortalidade infantil na América Latina são altas. Um estudo profundo de suas causas e aspectos variados em diferentes áreas só seria possível através de investigações continentais coordenadas, tais como a "Investigação Interamericana de Mortalidade em Adultos", já realizada pela OPAS. Esta mesma Organização Panamericana da Saúde está realizando uma investigação denominada "Investigação Interamericana de Mortalidade na Infância", em 13 áreas da América Latina, para investigar mortes de crianças menores de $\mathbf{5}$ anos. Tais mortes são estudadas por meio de entrevistas com as famílias, médicos e hospitais que cuidaram da criança durante a doença que ocasionou sua morte. Assim, obtém-se dados e informaçōes referentes às causas básicas e associadas de morte, ao mesmo tempo que se faz uma avaliação do estado de nutrição das crianças. Ao mesmo tempo, faz-se uma investigação entre as crianças vivas menores de 5 anos, utilizando-se uma técnica de amostragem probabilistica nos domicílios da área de estudo. São Paulo participa dessa Investigação por intermédio do Departamento de Estatística Aplicada da Faculdade de Higiene e Saúde Pública da Univesidade de São Paulo. A área em questão inclui o distrito da Capital, que possui $93 \%$ da população do Município.
\end{abstract}

A mortalidade infantil no município de Sáo Paulo é bastante alta, chegando em 1967 a apresentar o coeficiente de 73 óbitos de menores de um ano para mil nascidos vivos, número êsse que comparado ao de alguns países como, por exemplo, a Suécia (aproximadamente 13 por mil nascidos vivos) e Estados Unidos (aproximadamente 25 por mil nascidos vivos), carece de qualquer comentário a respeito.

$\mathrm{Em}$ trabalho anterior analisamos as principais causas de mortalidade infantil e sua tendência no município de São
Paulo ${ }^{2}$. Uma análise dêsse tipo, lògicamente, só pode ser feita através das estatísticas de mortalidade, cuja fonte de dados é o atestado de óbito. Esses dados, sabemos, são bastante precários, pois a qualidade dos atestados de óbito, entre nós, deixa muito a desejar ${ }^{1}$.

Dessa forma, uma análise das principais causas de morte, em qualquer grupo etário não traduz rigorosamente o que realmente está ocorrendo. 0 ideal seria então analisar os óbitos ocorridos através da história clínica, dos exames subsi.

Recebido para publicação em 27-8-1969.

(1) Do Departamento de Estatística Aplícada da Faculdade de Higiene e Saúde Pública da Universidade de São Paulo, São Paulo - Brasil. Colaborador Principal da Investigação Interamericana de Mortalidade na Infância, em São Paulo. 
LAURENTI, R. - A investigação inter-americana de mortalidade na infância em São Paulo, Brasil. Rev. Saúde públ., S. Paulo, 3(2):225-229, dez. 1969.

diários, das autópsias, quando feitas, etc., para se conhecer realmente qual a verda. deira causa básica da morte, assim como as causas associadas.

No que se refere à mortalidade infantil e à mortalidade de crianças menores de 5 anos, a oportunidade de um estudo dêsse tipo surgiu-nos agora com o convite para integrar um Estudo Internacional, de que São Paulo participa através da Faculdade de Higiene e Saúde Pública, por meio de seu Departamento de Estatística Aplicada. Tal estudo é a chamada "Investigação Interamericana de Mortalidade na Infância".

Já anteriormente foi realizada em São Paulo a "Investigação Interamericana de Mortalidade em Adultos", nos anos de 1962 a 1964, juntamente com outras cidades das Américas e uma da Europa. Essa Investigação demonstrou o valor de estudos continentais coordenados, evidenciando que cada área ou cidade tem suas características próprias de mortalidade nos adultos. Na citada "Investigação", os óbitos ocorridos no período eram estudados com visitas domiciliares à casa do falecido, visita a médicos, hospitais, clínicas, etc., para se obterem tôdas as informaçóes possíveis, a fim de se chegar a uma real causa básica da morte. Os resultados dêşe estudo já estão publicados ".

Um dos objetivos da Carta de Punta del Este ${ }^{5}$ consistiu em "reduzir a mortalidade dos menores de 5 anos de idade, pelo menos à metade dos coeficientes atuais". Segundo Puffer ${ }^{3}$, "por essas razōes foi proposto que se levassem a cabo investigações coordenadas no Continente, com o objetivo de melhor compreendermos os problemas que afetam a infância nas zonas urbanas e rurais das Américas." A realização de investigações coordenadas dessa índole foi também recomendada pelos colaboradores principais na "Investigação de Mortalidade de Adultos".

\section{OBJETIVOS E PROCEDIMENTOS}

Transcrevemos os objetivos e procedimentos, como se acham descritos em " $\mathrm{Fa}$ ses iniciales de la Investigación Interamericana de Mortalidad en la Niñez" 3.

"O objetivo geral da Investigação proposta é realizar uma Investigação Interamericana de Mortalidade na Infância, em comunidades selecionadas para obter coeficientes de Mortalidade corresponden. tes aos menores de 1 ano e os de 1 a 5 anos, que sejam os mais exatos e comparáveis possíveis, levando-se em conta fatôres nutritivos, sociais e ambientais. Com o fim de poder analisar as causas de morte, básica e associadas, e de estudar a interrelação entre doenças infecciosas, estados de deficiência nutritiva, fatôres sociais e outros, serão coletados dados completos sôbre os óbitos de menores de 5 anos de idade, de acôrdo com definições e procedimentos padronizados. Para estudar e comparar as diferenças biológicas e sociais daqueles que falecem nos primeiros anos de vida e daqueles que vivem, serão coletados também dados sôbre crianças vivas menores de 5 anos de idade, mediante uma amostragem probabilística de domicílios e de crianças nesse grupo etário.

Tanto os falecidos, como as crianças vivas, menores de 5 anos serão investigados mediante entrevistas com os familiares no domicílio e, nos casos de óbitos, serão feitas entrevistas a hospitais, clínicas, médicos, etc., a fim de se obterem dados completos da doença além de resultados de exames complementares, autópsias, etc.

Para se avaliarem os fatôres que causam a morte dessas crianças, a informação que se reunirá compreende a história da gravidez, o estado da criança ao nascer, a lactação, o crescimento e o desenvolvimento, assim como as condições sociais e ambientais.

Em cada uma das áreas selecionadas para participar da "Investigação", serão investigados, por ano, de 650 a 2.000 
LAURENTT, R. - A investigação inter-americana de mortalidade na infância em São Paulo, Brasil. Rev. Saúde publ., S. Paulo, 3(2):225-229, dez. 1969.

óbitos durante 2 anos (24, meses consecutivos). Em muitas áreas serão incluídas tanto zonas rurais como urbanas.

0 número de óbitos que será investigado em cada área dependerá, em parte, da magnitude do coeficiente de mortalidade e, em parte, da densidade populacional. Serão incluídas áreas de grande altitude, como também aquelas em que existem sérios problemas nutricionais.

Nas áreas de estudo se reunirão, pelo método da amostragem probabilística, informações semelhantes sôbre a história da gravidez, estado da criança ao nascer, lactação, crescimento e desenvolvimento e condiçōes sociais e ambientais de um grupo de crianças vivas, menores de 5 anos. No caso de estudo de crianças vivas, o período de dois anos será dividido em 24 períodos de um mês, e, em cada um dêles, será tomada uma amostra de domicílios e crianças vivas. Serão utilizadas técnicas precisas de amostragem probabilística para selecionar os domicílios e preencher os questionários de todos os menores de 5 anos de idade. Em cada ano de estudo serão investigados de 650 a 1.000 casos de crianças vivas.

Com o objetivo de que o programa da investigação beneficie tanto os programas de Ensino Médico como os de Saúde Pública, os projetos serão desenvolvidos em Escolas de Medicina ou nas Escolas de Saúde Pública, com a colaboração dos Serviços de Saúde".

\section{AS AREAS DE ESTUDO}

Foram selecionadas 13 áreas da América Latina para serem incluídas na Investigação e também uma área dos Estados Unidos (San Francisco, California).

As áreas da "Investigação" na América Latina são as seguinte: 1) El Chaco (Argentina) ; 2) San Juan (Argentina); 3) La Paz (Bolívia); 4) Cali (Colômbia) ; 5) Cartagena (Colômbia) ; 6) Medellin (Colômbia); 7) Kingston (Jamaica) ; 8) Monterrey (México); 9) San- tiago (Chile); 10) Ribeirão Prêto (Brasil); 11) Recife (Brasil) ; 12) São Paulo (Brasil); 13) El Salvador (San Salvador).

\section{A INVESTIGAÇÃO NA AREA DE SAO PAULO}

A área escolhida para a "Investigação de Mortalidade na Infância" em São Paulo é o diștrito da Capital, que compreende $93 \%$ da populaçáo do município da capital do Estado, contando com 48 sub-distritos.

A Investigação em São Paulo foi iniciada em junho de 1968 e uma descrição rápida da metodologia empregada é a que se segue:

Mensalmente (nos primeiros dez dias do mês) os cartórios do Registro Civil dos 48 sub-distritos enviam ao Departamento de Estatística do Estado os atestados de óbitos ocorridos no mês anterior. Separamos numa primeira fase todos os atestados de crianças menores de 5 anos e dêsses separamos aqueles cuja residência é fora da área de estudo (pois nosso ob-jetivo é o estudo da mortalidade em menores de 5 anos, residentes no distrito da Capital). A seguir, é realizado um sorteio probabilístico, aplicando-se uma amostragem sistemática, que nos dará mensalmente um determinado número de casos a investigar, e o tamanho total da amostra; o tamanho total da amostra proposta por ano é de 2.000 casos.

Feito o sorteio mensal, os atestados de óbito primeiramente são entregues a educadoras sanitárias treinadas em técnicas de entrevistas, as quais vão à residência dos familiares da criança falecida para preencher um questionário (igual ao usado em outras áreas onde se procede a investigação). Nesse questionário existem ítens para a caracterização da habitação, da composição familiar, da ocupaçấo dos membros da família, dos óbitos ocorridos na casa no último ano, gestações que teve a mãe da criança, assistência pré-natal na gestação da criạnça 
LAURENTI, R. - A investigação inter-americana de mortalidade na infåncia em São Paulo, Brasil. Rev. Saúde públ., S. Paulo, 3(2):225-229, dez. 1969.

falecida, problemas da gravidez e parto, local de nascimento da criança (casa, hospital, etc.), amamentação da criança, idade em que foram introduzidos outros alimentos especificados, vacinação que a criança recebeu, assim como outros fatôres. Pergunta-se também o nome do médico, clínica ou hospital em que a criança estêve em tratamento, não só na doença que causou a morte, mas também por outras causas. Finalmente, a entrevistadora pede à mãe (ou outro informante) que descreva a doença que a criança teve.

Preenchida essa primeira parte do questionário e com os dados obtidos, um médico pediatra, membro da equipe da Investigação, vai tentar obter os dados referentes à história clínica, exame físico, exame de laboratório, autópsia, se houver, enfim tudo o que foi feito do ponto de vista de assistência médica à criança falecida. Nos casos em que ocorreu um falecimento sem assistência médico-hospitalar, o próprio pediatra irá fazer uma entrevista familiar, para obter o maior número possível de informações junto à mãe, para se tentar chegar a uma conclusão sôbre a doença. Obtidas tôdas essas informaçōes é feito um resumo e chega-se a uma conclusão sôbre a causa básica da morte e as causas associadas, procurando-se também, sempre que possível, fazer uma avaliação do estado de nutrição da criança.

Paralelamente, são feitos levantamentos mensais em todos os cartórios para se obter com exatidão o número de nascidos vivos, que foram registrados e que são residentes na área de estudo; investiga-se também se as crianças falecidas, menores de 1 ano têm registro de nascimento, nos casos em que a família não sabe ou não tem certeza se a criança foi registrada é feita pesquisa nos cartórios. Com isso, o que se procura é obter os dados para se medir com exatidão a magnitude do coeficiente de mortalidade infantil.

Os questionários preenchidos e completos são periòdicamente enviados à $\mathrm{Or}$ - ganização Sanitária Pan-Americana, em Washington, onde são revistos e classificados, juntamente com os questionários procedentes de outras áreas de estudo.

Em São Paulo, desde o início da Investigação, temos contado com o apoio de todo o pessoal médico entrevistado, assim como dos hospitais visitados, onde todos os pesquisadores têm sido recebidos com a máxima boa vontade pelo pessoal dos Serviços de Arquivo Médico e Estatística. Algumas dificuldades que surgiram foram paulatinamente vencidas, de tal forma que os resultados que estamos obtendo são bastante compensadores.

Além do estudo dos óbitos, é feita também pela educadora sanitária a entrevista familiar nos domicílios sorteados pela amostragem probabilística, a fim de se obterem dados de crianças vivas menores de 5 anos, residentes na área de estudo.

A equipe da Investigação em São Paulo conta com um colaborador principal, 3 médicos pediatras, 5 alunos sexto-anistas de medicina, quatro educadoras sanitárias, um técnico, um auxiliar e dois agentes de campo em amostragem, além de secretária e pessoal auxiliar (coletadores de dàdos em cartório e datilógrafos).

\section{RESULTADOS QUE SERAO OBTIDOS NA INVESTIGACAO}

Após o término da Investigação, os resultados evidenciarão a magnitude real da mortalidade infantil nas áreas de estudo, assim como as reais causas de morte, nesse grupo etário e no grupo de um a 5 anos de idade. Por outro lado, serão obtidos dados para a análise dos fatôres associados e que contribuiram para as causas de morte, tais como sócio-econômico, assistência médico-hospitalar e principalmente informações sôbre o estado nutritivo das crianças. $O$ que se espera é conhecer o quanto a desnutrição é responsável, quer como causa básica, quer como associada, da mortalidade. Esse dado é muito importante, visto que a des- 
LAURENTT, R. - A investigação inter-americana de mortalidade na infância em São Paulo, Brasil. Rev. Saúde públ., S. Paulo, 3(2):225-229, dez. 1969.

nutrição raramente aparece nos atestados de óbito como causa de morte e sua medida não é conhecida, embora se tenha a idéia de que exista, e em algumas áreas é um fator importante na mortalidade.

Por outro lado, como também serão investigadas crianças vivas, poder-se-ão fazer comparações sôbre fatôres mencionados nestas e naquelas falecidas.

Outras informações de interêsse e que serão conhecidas, dizem respeito aos dados vitais, pois serão medidos, entre outros, registros de nascimento, quer na amostragem de crianças vivas, quer o sub-registro de nascimento de falecidos menores de 1 ano.

As informações obtidas em cada área mostrarão problemas específicos e a comparação dos resultados permitirá evidenciar diferenças mostrando se cada área de estudo possui características próprias de mortalidade nesse grupo etário, como foi observado no estudo na "Investigação de Mortalidade em Adultos"."

LAURENTI, R. - Interamerican investigation of childhood mortality in the city of São Paulo, Brazil. Rev. Saride puibl., S. Paulo, 3(2) :225-229, dez. 1969.

SUMMARY - Infant mortality rates in Latin America are high. A thorough study of its causes and different aspects in several areas would be feasible only through coordinate continental investigations such as the "Inter American Investigation of Mortality in Adults". The Pan American Health Organization is carrying out a study named "Interamerican Investigation of Childhood Mortality", in 13 areas of Latin America, to investigate deaths of children of less than 5 years of age. These deaths are studied through interviews with the families, doctors and hospitals which were in contact with the children during the period of illness that led to death. Thus, data and information are obtained, with relation to the basic and related causes of death, as well as an evaluation of the nutritional state of the children. Simultaneously, an investigation is made on alive children of less than five years of age, through a random sampling technique of the houses in the area of study. São Paulo participates in this Investigation through the Department of Applied Statistics of the University of São Paulo School of Public Health. The area of study includes the district of the Capital, which contains $93 \%$ of the population of the entire county.

\section{REFERENCIAS BIBLIOGRAFICAS}

1. MILANESI, M. L. \& LAURENTI, R. O estudo interamericano de mortalidade em Sáo Paulo. I - Estudo atual da certificação médica da causa de óbito no distrito da Capital. Rev. Ass. med. bras., 10:111-116, maio-jun. 1964.

2. MILANESI, M. L. \& LAURENTI, R. Mortalidade infantil no município de São Paulo. Análise de seu comportamento nos últimos 15 anos. Rev Saúde públ., S. Paulo, 1:44-50, jun. 1967.

3. PUFFER, R. R. - Fases iniciales de la "Investigación Interamericana de Mortalidad en la Niñez". Bol. Ofic. Sanit. panamer., 16:114-126, ago. 1968.

4. PUFFER, R. R. \& GRIFFITH, G. W. Patterns of urban mortality. Washington, D.C., Pan American Health Organization, 1967. (Scientific Publ. 151).

5. UNION PANAMERICANA. Alianza para el Progresso. Washington, D.C., 1961 (OEA/Ser. H/XII.1). 